\title{
Six-qubit two-photon hyperentangled cluster states: characterization and application to quantum computation
}

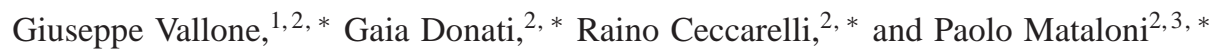 \\ ${ }^{1}$ Museo Storico della Fisica e Centro Studi e Ricerche Enrico Fermi, \\ Via Panisperna 89/A, Compendio del Viminale, Roma 00184, Italy \\ ${ }^{2}$ Dipartimento di Fisica, Università Sapienza di Roma, Roma 00185, Italy \\ ${ }^{3}$ Istituto Nazionale di Ottica Applicata (INOA-CNR), L.go E. Fermi 6, 50125 Florence, Italy
}

(Dated: October 27, 2018)

\begin{abstract}
Six-qubit cluster states built on the simultaneous entanglement of two photons in three independent degrees of freedom, i.e. polarization and a double longitudinal momentum, have been recently demonstrated. We present here the peculiar entanglement properties of the linear cluster state $\left|\widetilde{\mathrm{LC}}_{6}\right\rangle$ related to the three degrees of freedom. This state has been adopted to realize various kinds of Controlled NOT (CNOT) gates, obtaining in all the cases high values of the gate fidelity. Our results demonstrate that a number of qubits $\leq 10$ in cluster states of two photons entangled in multiple degrees of freedom is achievable. Furthermore, these states represent a promising approach towards scalable quantum computation in a medium term time scale. The future perspectives of a hybrid approach to one-way quantum computing based on multi-degree of freedom and multi-photon cluster states are also discussed in the conclusions of this paper.
\end{abstract}

PACS numbers:

\section{INTRODUCTION}

Multiqubit graph states [1] are a basic resource for a number of important quantum information applications. These states have been proposed in particular for advanced tests of quantum nonlocality in which the violation of local realism increases exponentially with the number of qubits [2, 3, 4, 5], and for the realization of quantum computation algorithms of increasing complexity in the one-way model [6, 7]. Other application fields deal with quantum communication [8] and quantum error correction [9].

In recent years, photon cluster states of four, six and up to ten qubits have been realized by different approaches and used to deeply investigate the peculiar properties of high dimensional entanglement [10] and to perform basic quantum computation algorithms [11, 12].

Two strategies are generally used to create multiqubit cluster states: one consists of increasing the number of entangled photons [13, 14, 15, 16, 17], the second one is based on the encoding of more qubits in different degrees of freedom of the particles [11, 12, 18, 19]. By the first approach, some examples of four and six photon [13, 14, 15, 16] cluster states have been experimentally demonstrated, up to now, with very low rates. The second approach, which is based on two-photon hyperentanglement, has been used to create two-photon fourqubit cluster states [18, 19, 20, 21, 22, 23, 24, 25, 26, 27, 28]. By using hyperentanglement, five photons have been recently entangled in ten qubits encoded in the polarization and longitudinal momentum degrees of freedom (DOFs) [19].

The advantages of the hyperentangled state approach, as far as generation/detection rate and fidelity of the states are concerned, have been already demonstrated [11, 12]. These properties have been very recently confirmed by the realiza-

*URL: http://quantumoptics.phys.uniroma1.it/
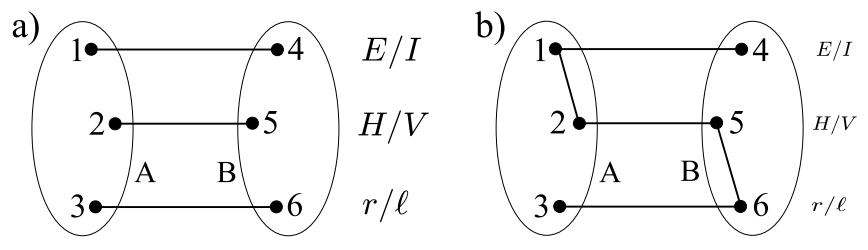

FIG. 1: (a) Graph associated to the hyperentangled state $\left|\mathrm{HE}_{6}\right\rangle$. Each set represents a photon and every vertex is associated to a qubit. Qubits 1 and 4 are encoded in the $E / I$ DOF, qubits 2 and 5 in polarization and qubits 3 and 6 in the $r / \ell$ DOF. See text for further details. (b) Graph associated to the two-photon six-qubit linear cluster state $\left|\mathrm{LC}_{6}\right\rangle .\left|\mathrm{LC}_{6}\right\rangle$ can be obtained from $\left|\mathrm{HE}_{6}\right\rangle$ by application of two $\mathrm{CZ}$ operations between qubits belonging to different DOFs.

tion of the linear 2-photon 6-qubit cluster state $\left|\widetilde{\mathrm{LC}}_{6}\right\rangle$ starting from the triple entanglement of two photons in three independent DOFs [29], namely the polarization and a double longitudinal momentum. The $\left|\widetilde{\mathrm{LC}}_{6}\right\rangle$ is the only distribution of six qubits between two particles whose perfect correlations have the same nonlocality as those of the six-qubit GreenbergerHorne-Zeilinger state [5], but only requires two separated carriers [4].

In this paper we give a detailed characterization of the $\left|\widetilde{\mathrm{LC}}_{6}\right\rangle$ state realized by using the triple hyperentanglement of two photons and demonstrate its feasibility for one-way quantum computation by the high fidelity realization of different kinds of CNOT gates.

The paper is organized as follows. In Sec. II we describe the realization of the six-qubit linear cluster state, derived from the application of suitable CPHASE gates to a six-qubit hyperentangled state. Sec. IIIreports on the characterization of the $\left|\widetilde{\mathrm{LC}}_{6}\right\rangle$ state by a sequence of quantum tomographic reconstructions performed in the three DOFs. Sec. IV de- 
scribes how the CNOT gate has been efficiently realized with six qubits. Finally, the future perspectives of the realization of multiqubit cluster states built on an increasing number of photon DOFs are discussed in the conclusions of Sec. $\mathrm{V}$

\section{GENERATION OF THE SIX-QUBIT CLUSTER STATE}

Cluster states are peculiar entangled states associated to $n$ dimensional lattices where each vertex $i$ represents a qubit and connections between vertices correspond to Ising interactions between the two-level quantum systems. Two-dimensional lattices have proved to be a universal resource for Quantum Computation (QC) [6]; from here on, we shall then restrict ourselves to the case $n=2$. The explicit expression of a cluster state is obtained by preparation of each qubit in the state $|+\rangle_{i}=\frac{1}{\sqrt{2}}\left(|0\rangle_{i}+|1\rangle_{i}\right)$ and subsequent application of a CPHASE gate, $\mathrm{CZ}_{i j}$, between two adjacent vertices $i$ and $j$. We have

$$
\mathrm{CZ}_{i j}=|0\rangle_{i}\left\langle 0\left|\otimes \mathbb{1}_{j}+\right| 1\right\rangle_{i}\langle 1| \otimes Z_{j},
$$

where $\mathbb{1}$ is the identity operator. From now we will use the following simplified notation for the Pauli operators: $\sigma_{z}^{(i)} \equiv$ $Z_{i}$ and analogous relations for $\sigma_{x}^{(i)}$ and $\sigma_{y}^{(i)}$.

For a lattice $\mathcal{L}$ with $N$ sites, the corresponding cluster state can then be written as

$$
\left|\Phi_{N}^{\mathcal{L}}\right\rangle=\left(\prod_{i, j \text { linked }} \mathrm{CZ} Z_{i j}\right)|+\rangle^{N}
$$

where $|+\rangle^{N}=|+\rangle_{1} \otimes|+\rangle_{2} \otimes \ldots \otimes|+\rangle_{N}$.

In general, the cluster state associated to a specific graph can be equivalently defined as the only state satisfying the eigenvalue equations

$$
g_{i}\left|\Phi_{N}^{\mathcal{L}}\right\rangle=\left|\Phi_{N}^{\mathcal{L}}\right\rangle
$$

for every lattice vertex $i$, where the operators

$$
g_{i}=X_{i} \bigotimes_{j \in \mathcal{N}_{i}} Z_{j}
$$

are known as the stabilizer generators for the cluster state. $\mathcal{N}_{i}$ is the set of vertices connected with the vertex $i$.
The linear cluster state $\left|\mathrm{LC}_{6}\right\rangle$ is the state associated to the lattice shown in Fig. 1(b). We generated a six-qubit twophoton linear cluster state $\left|\widetilde{\mathrm{LC}}_{6}\right\rangle$, equivalent to $\left|\mathrm{LC}_{6}\right\rangle$ up to single qubit unitary transformations, starting from the hyperentangled state $\left|\widetilde{\mathrm{HE}}_{6}\right\rangle$ and exploiting the three degrees of freedom (DOFs) of polarization and two different kinds of longitudinal momentum. To show that the cluster state $\left|\widetilde{\mathrm{LC}}_{6}\right\rangle$ obtained in the laboratory is equivalent to $\left|\mathrm{LC}_{6}\right\rangle$, we start describing the source of the hyperentangled state $\left|\widetilde{\mathrm{HE}}_{6}\right\rangle$, the first step for the generation of the linear cluster $\left|\widetilde{\mathrm{LC}}_{6}\right\rangle$.

The two-photon six-qubit source, extensively described elsewhere [22, 23, 29], consists of a continuous wave (cw), vertically-polarized $\mathrm{Ar}^{+}$laser beam $\left(P=50 \mathrm{~mW}, \lambda_{p}=\right.$ $364 \mathrm{~nm}$ ) interacting through spontaneous parametric downconversion (SPDC) with a Type I, $0.5 \mathrm{~mm}$ thick $\beta$-BariumBorate (BBO) crystal. The nonlinear interaction between the laser beam and the $\mathrm{BBO}$ crystal produces degenerate photon pairs at wavelength $\lambda=728 \mathrm{~nm}$, entangled in polarization and belonging to the surfaces of an emission cones. Referring to Fig. 2(a), the insertion of a holed mask allows us to select four pairs of correlated spatial modes from the conical surface, which is all we need for the creation of the hyperentangled state $\left|\widetilde{\mathrm{HE}}_{6}\right\rangle$. The labels used to identify the selected modes require some explanations [cfr. Fig. 2 (b)]: the distinction between left and right modes provides us with the first longitudinal momentum DOF $(r / \ell$, also known as the linear momentum $\mathbf{k}$ ), while distinguishing between external and internal modes supplies the second momentum DOF $(E / I)$. Moreover, the conical emission of the BBO crystal can be divided into an "up" circular half and a "down" one with respect to an ideal horizontal line passing through the center of the mask. Every mode belonging to the "up" half shall be associated to carrier photon $A$; an analogous correspondence is adopted for the "down" half and the second carrier photon $B$. By doing so we have at our disposal two SPDC photons, $A$ and $B$, to each of which we associate three different qubits corresponding to the three DOFs (polarization, first, and second momentum) introduced above.

By appropriately setting the phase of each pair of modes, the source generates the hyperentangled state $\left|\widetilde{\mathrm{HE}}_{6}\right\rangle$, explicitly written as

$$
\begin{aligned}
\left|\widetilde{H E}_{6}\right\rangle & =\frac{1}{\sqrt{2}}\left(|H H\rangle_{A B}-|V V\rangle_{A B}\right) \otimes \frac{1}{2}\left(|E r\rangle_{A}|E \ell\rangle_{B}+|E \ell\rangle_{A}|E r\rangle_{B}+|I r\rangle_{A}|I \ell\rangle_{B}+|I \ell\rangle_{A}|I r\rangle_{B}\right)= \\
& =\frac{1}{\sqrt{2}}\left(|E E\rangle_{A B}+|I I\rangle_{A B}\right) \otimes \frac{1}{\sqrt{2}}\left(|H H\rangle_{A B}-|V V\rangle_{A B}\right) \otimes \frac{1}{\sqrt{2}}\left(|r \ell\rangle_{A B}+|\ell r\rangle_{A B}\right)
\end{aligned}
$$

It comes out that the state $\left|\widetilde{\mathrm{HE}}_{6}\right\rangle$ is given by a tensor product of three maximally entangled state, one for each DOF.
By setting the following correspondences between physical 
(a)
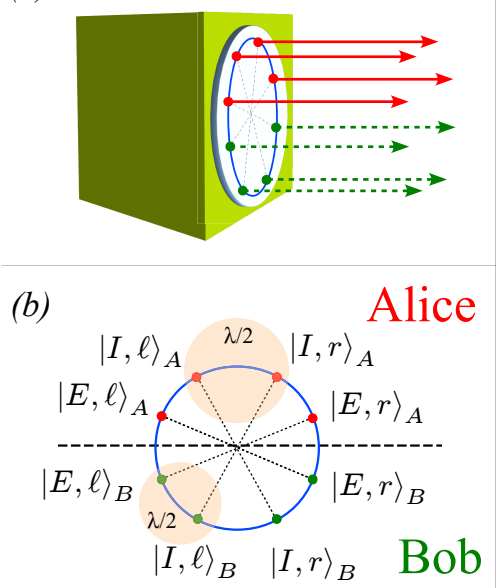

(c)

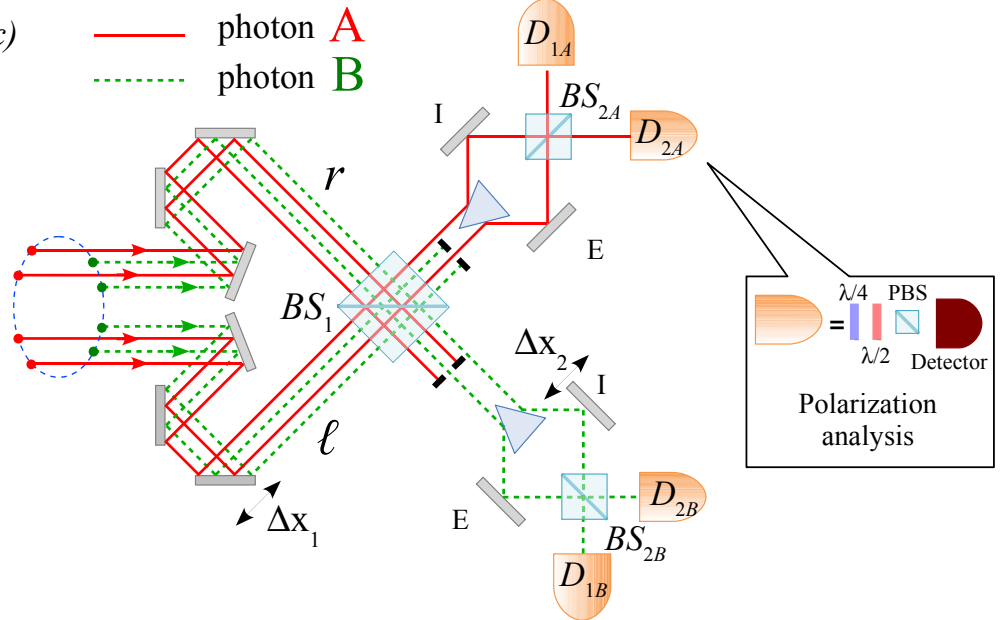

FIG. 2: Setup of the experiment. (a) Source (green box) of the 8-mode hyperentangled state. A detailed description of the source is given in [22, 23, 29]. (b) Mode labelling: upper (lower) modes correspond to Alice (Bob) photon. For each photon we indicate with $|r\rangle(|\ell\rangle)$ the right (left) modes and with $|I\rangle(|E\rangle)$ the internal (external) modes. We also show the two half wave plates $(\lambda / 2)$ used to transform the hyperentangled state $\left|\widetilde{\mathrm{HE}}_{6}\right\rangle$ to the cluster state $\left|\widetilde{\mathrm{LC}}_{6}\right\rangle$. The $\lambda / 2$ on the $I$ modes of photon $A$ is oriented at $45^{\circ}$ while the $\lambda / 2$ on the $\ell$ modes of photon $B$ is oriented at $0^{\circ}$. (c) Measurement scheme: the momentum measurement setup consists of two chained interferometers, the first ( $\left.B S_{1}\right)$ measuring the $r / \ell$ qubit, while the second $\left(B S_{2 A}\right.$ and $\left.B S_{2 B}\right)$ measuring the $I / E$ qubit. Polarization analysis is performed by standard waveplates and polarizing beam splitters (PBS). Path delays $\Delta x_{1}$ and $\Delta x_{2}$, are varied to obtain the optimal temporal superposition of the modes respectively in the first and second interferometer.

and computational qubits,

$$
\begin{aligned}
\left\{|E\rangle_{A},|I\rangle_{A}\right\} & \rightarrow\left\{|0\rangle_{1},|1\rangle_{1}\right\}, \\
\left\{|H\rangle_{A},|V\rangle_{A}\right\} & \rightarrow\left\{|0\rangle_{2},|1\rangle_{2}\right\}, \\
\left\{|r\rangle_{A},|\ell\rangle_{A}\right\} & \rightarrow\left\{|0\rangle_{3},|1\rangle_{3}\right\}, \\
\left\{|E\rangle_{B},|I\rangle_{B}\right\} & \rightarrow\left\{|0\rangle_{4},|1\rangle_{4}\right\}, \\
\left\{|H\rangle_{B},|V\rangle_{B}\right\} & \rightarrow\left\{|0\rangle_{5},|1\rangle_{5}\right\}, \\
\left\{|r\rangle_{B},|\ell\rangle_{B}\right\} & \rightarrow\left\{|0\rangle_{6},|1\rangle_{6}\right\},
\end{aligned}
$$

we can express the state [5] as

$$
\left|\widetilde{\mathrm{HE}}_{6}\right\rangle=\mathrm{H}_{2} X_{3} \mathrm{H}_{3} \mathrm{H}_{4} Z_{5}\left|\mathrm{HE}_{6}\right\rangle,
$$

where $\left|\mathrm{HE}_{6}\right\rangle$ is the state associated to the graph shown in Fig. 11 a) and $\mathrm{H}_{i}$ is the Hadamard operator acting on qubit $i$. From the definition of graph states in eq. (2), $\left|\mathrm{LC}_{6}\right\rangle$ is obtained from the graph state $\left|\mathrm{HE}_{6}\right\rangle$ by the application of the two-qubit gates $\mathrm{CZ}_{12}$ and $\mathrm{CZ}_{56}$.

We build the state $\left|\widetilde{\mathrm{LC}}_{6}\right\rangle$ by applying the gates $\mathrm{CX}_{12}$ and $\mathrm{CZ}_{56}$ to the hyperentangled state $\left|\mathrm{HE}_{6}\right\rangle$. The gate $\mathrm{CX}$ is defined as $\mathrm{CX}_{i j}=|0\rangle_{i}\left\langle 0\left|\otimes \mathbb{1}_{j}+\right| 1\right\rangle_{i}\langle 1| \otimes X_{j}$. We are now in the position to state the relation between the state $\left|\widetilde{\mathrm{LC}}_{6}\right\rangle$, and the state $\left|\mathrm{LC}_{6}\right\rangle$ :

$$
\begin{aligned}
\left|\widetilde{\mathrm{LC}}_{6}\right\rangle & =\mathrm{CX}_{12} \mathrm{CZ}_{65}\left|\widetilde{\mathrm{HE}}_{6}\right\rangle \\
& =\mathrm{CX}_{12} \mathrm{CZ}_{65}\left(\mathrm{H}_{2} X_{3} \mathrm{H}_{3} \mathrm{H}_{4} Z_{5}\right)\left|\mathrm{HE}_{6}\right\rangle \\
& =\left(\mathrm{H}_{2} X_{3} \mathrm{H}_{3} \mathrm{H}_{4} Z_{5}\right) \mathrm{CZ}_{12} \mathrm{CZ}_{65}\left|\mathrm{HE}_{6}\right\rangle \\
& =\mathrm{H}_{2} X_{3} \mathrm{H}_{3} \mathrm{H}_{4} Z_{5}\left|\mathrm{LC}_{6}\right\rangle .
\end{aligned}
$$

The previous relations can be easily demonstrated by using the property $\mathrm{CX}_{i j} \mathrm{H}_{j}=\mathrm{H}_{j} \mathrm{CZ}_{i j}$. We thus see that the generated cluster state $\left|\widetilde{\mathrm{LC}}_{6}\right\rangle$ is equivalent to the linear six-qubit twophoton cluster state $\left|\mathrm{LC}_{6}\right\rangle$ up to the unitary transformation $\left[\mathrm{H}_{2} \mathrm{X}_{3} \mathrm{H}_{3} \mathrm{H}_{4} Z_{5}\right]$ consisting of single qubit unitaries. In the generated state, qubits 1 and 4 are encoded in the $E / I$ longitudinal momentum DOF, qubits 2 and 5 in the polarization variable and qubits 3 and 6 in the $r / \ell$ momentum DOF (see Fig. 11. Specifically, the relation given in (8) between $\left|\widetilde{\mathrm{LC}}_{6}\right\rangle$ and $\left|\mathrm{LC}_{6}\right\rangle$ implies that $\left|\widetilde{\mathrm{LC}}_{6}\right\rangle$ is the only common eigenstate of the generators $\left\{\widetilde{g}_{i}\right\}$ obtained from $\left\{g_{i}\right\}$ by changing $X_{2} \leftrightarrow Z_{2}$, $X_{3} \rightarrow-Z_{3}, Z_{3} \rightarrow X_{3}, X_{4} \leftrightarrow Z_{4}$ and $X_{5} \rightarrow-X_{5}$.

Starting from Eq. (8), we can write the following explicit expressions for the generated state $\left|\widetilde{\mathrm{LC}}_{6}\right\rangle$ by differently factoring the terms referring to the three considered DOFs: 


$$
\begin{aligned}
\left|\widetilde{\mathrm{LC}}_{6}\right\rangle & =\frac{1}{2}\left[|E E\rangle\left|\phi^{+}\right\rangle_{\pi}|r \ell\rangle+|E E\rangle\left|\phi^{-}\right\rangle_{\pi}|\ell r\rangle+|I I\rangle\left|\psi^{+}\right\rangle_{\pi}|r \ell\rangle-|I I\rangle\left|\psi^{-}\right\rangle_{\pi}|\ell r\rangle\right]= \\
& =\frac{1}{2}\left[|E E\rangle|H H\rangle\left|\psi^{+}\right\rangle_{\mathbf{k}}+|E E\rangle|V V\rangle\left|\psi^{-}\right\rangle_{\mathbf{k}}+|I I\rangle|V H\rangle\left|\psi^{+}\right\rangle_{\mathbf{k}}+|I I\rangle|H V\rangle\left|\psi^{-}\right\rangle_{\mathbf{k}}\right]= \\
& =\frac{1}{2}\left[\left|\phi^{+}\right\rangle_{c}|++\rangle|r \ell\rangle+\left|\phi^{-}\right\rangle_{c}|--\rangle|r \ell\rangle+\left|\phi^{+}\right\rangle_{c}|+-\rangle|\ell r\rangle+\left|\phi^{-}\right\rangle_{c}|-+\rangle|\ell r\rangle\right],
\end{aligned}
$$

where we omitted the subscripts $A B$. The states $\left|\phi^{ \pm}\right\rangle_{\pi}=$ $\frac{1}{\sqrt{2}}\left(|H H\rangle_{A B} \pm|V V\rangle_{A B}\right)$ and $\left|\psi^{ \pm}\right\rangle_{\pi}=\frac{1}{\sqrt{2}}\left(|H V\rangle_{A B} \pm\right.$ $\left.|V H\rangle_{A B}\right)$ are the four polarization Bell states, while the states $\left|\psi^{ \pm}\right\rangle_{\mathbf{k}}$ and $\left|\phi^{ \pm}\right\rangle_{c}$ are the standard Bell states encoded in the $r / \ell$ and $E / I$ degrees of freedom, respectively (the " $c$ " subscript standing for "cone").

The realization of the two-qubit gates responsible for the transformation of the hyperentangled state $\left|\widetilde{\mathrm{HE}}_{6}\right\rangle$ into the cluster state $\left|\widetilde{\mathrm{LC}_{6}}\right\rangle$ in terms of optical components was made possible by the insertion of two wave-plates after the holed mask; since qubits 1 and 2 belong to photon $A$, the first $\mathrm{CX}_{12}$ gate was realized by means of a $\lambda / 2$ wave-plate oriented at $45^{\circ}$ and intercepting the two internal $A$ modes (see Fig. 2 b) and Eq. (6a)). Analogously, the $\mathrm{CZ}_{65}$ gate was obtained thanks to a second $\lambda / 2$ wave-plate oriented at $0^{\circ}$ and intercepting the two left $B$ modes (see Fig. 2 (b) and Eq. (6f) ). It actually proved convenient to have two separated $\lambda / 2$ waveplates on the left $B$ modes, but this was a choice uniquely related to our specific experimental setup.

\section{CHARACTERIZATION OF THE SIX-QUBIT CLUSTER STATE}

Let us refer to Fig. 22(c). The two chained interferometers, whose core elements are the three symmetric beam splitters $B S_{1}, B S_{2 A}$ and $B S_{2 B}$, allow the simultaneous measurement of the three single-qubit compatible observables associated to both particles $A$ and $B$. The $r$ modes are made indistinguishable (in space as well as in time) from the $\ell$ ones on $B S_{1}$, while $E$ and $I$ modes are matched on $B S_{2 A}$ or $B S_{2 B}$ depending on which photon they refer to. By means of a trombone mirror assembly in each of the two interferometers, it is possible to act on the optical path delays, $\Delta x_{1}$ and $\Delta x_{2}$, and find the optimal temporal superposition conditions for both of the interference phenomena. Let us now refer to the $B S_{1}$ : we set $\left\{|\ell\rangle_{j},|r\rangle_{j}\right\}$ and $\left\{\left|\ell^{\prime}\right\rangle_{j},\left|r^{\prime}\right\rangle_{j}\right\}$, for $j=A, B$, as its input and output states. The insertion of a thin glass plate intercepting two right $A$ modes (one internal and one external) transforms the input states in the following way: $\left|\phi_{A}\right\rangle_{\mathbf{k}}=\frac{1}{\sqrt{2}}\left(|\ell\rangle_{A}+e^{-i \phi_{A}}|r\rangle_{A}\right) \rightarrow\left|\ell^{\prime}\right\rangle_{A}$ and $\left|\phi_{A}^{\perp}\right\rangle_{\mathbf{k}}=\frac{1}{\sqrt{2}}\left(|\ell\rangle_{A}-e^{-i \phi_{A}}|r\rangle_{A}\right) \rightarrow\left|r^{\prime}\right\rangle_{A}$, for external and internal modes. By detecting the photons on the $\left|\ell^{\prime}\right\rangle$ or the $\left|r^{\prime}\right\rangle$ output we are projecting the input state respectively into $\left|\phi_{A}\right\rangle$ or $\left|\phi_{A}^{\perp}\right\rangle$. An analogous glass plate intercepts the left $B$ modes $^{1}$.

Two more such phase shifters, intercepting the external A and $\mathrm{B}$ modes, are inserted in the second interferometer before $B S_{2 A}$ and $B S_{2 B}$. Four single-photon detectors $D_{1 A}, D_{2 A}$, $D_{1 B}$ and $D_{2 B}$ receive the radiation belonging to the "up" and "down" output modes (see Fig. 2(b)), which we can label as $\left\{\left|E^{\prime}\right\rangle_{j},\left|I^{\prime}\right\rangle_{j}\right\}$ for $j=A, B$. In the presence of the glass plates cited above, the following input-output transformations concerning $B S_{2 A}$ and $B S_{2 B}$ hold: $|\delta\rangle_{c}=\frac{1}{\sqrt{2}}\left(e^{-i \delta}|E\rangle_{j}+\right.$ $\left.|I\rangle_{j}\right) \rightarrow\left|E^{\prime}\right\rangle_{j}$ and $\left|\delta^{\perp}\right\rangle_{c}=\frac{1}{\sqrt{2}}\left(e^{-i \delta}|E\rangle_{j}-|I\rangle_{j}\right) \rightarrow\left|I^{\prime}\right\rangle_{j}$. Finally, a polarization analyzer constituted of a $\lambda / 2$ waveplate, a $\lambda / 4$ wave-plate and a polarizing beam splitter (PBS) is added in front of each detector. In these conditions, we recorded nearly 500 coincidences per second.

The characterization of the generated state $\left|\widetilde{\mathrm{LC}}_{6}\right\rangle$ relies on a tomographic reconstruction technique followed by a "maximum likelihood" method [30]. Particularly, we aim at recovering Eq. 9), which shows three alternative and perfectly equivalent ways of writing the cluster state $\left|\widetilde{\mathrm{LC}}_{6}\right\rangle$. Indeed, Eq. (9) is important to prove since it highlights the inner structure of the generated state. As we see, each of the expressions (9) is obtained by writing the states of four qubits corresponding

TABLE I: Fidelities of Bell states for each DOF. We selected a separable state for two DOFs (first column) and performed a tomographic reconstruction of the density matrix of the remaining DOF (second column). The expected output states and the relative fidelities are shown in the last two columns.

\begin{tabular}{|c|ccc|}
\hline \hline separable basis & output DOF & output state & Fidelity \\
\hline$|E E\rangle_{c}|r \ell\rangle_{\mathbf{k}}$ & $\pi$ & $\left|\phi^{+}\right\rangle_{\pi}$ & $0.821 \pm 0.014$ \\
$|E E\rangle_{c}|\ell r\rangle_{\mathbf{k}}$ & & $\left|\phi^{-}\right\rangle_{\pi}$ & $0.917 \pm 0.017$ \\
$|I I\rangle_{c}|r \ell\rangle_{\mathbf{k}}$ & & $\left|\psi^{+}\right\rangle_{\pi}$ & $0.905 \pm 0.013$ \\
$|I I\rangle_{c}|\ell r\rangle_{\mathbf{k}}$ & & $\left|\psi^{-}\right\rangle_{\pi}$ & $0.828 \pm 0.025$ \\
\hline$|E E\rangle_{c}|H H\rangle_{\pi}$ & $r / \ell$ & $\left|\psi^{+}\right\rangle_{\mathbf{k}}$ & $0.897 \pm 0.008$ \\
$|E E\rangle_{c}|V V\rangle_{\pi}$ & & $\left|\psi^{-}\right\rangle_{\mathbf{k}}$ & $0.933 \pm 0.016$ \\
$|I I\rangle_{c}|V H\rangle_{\pi}$ & & $\left|\psi^{+}\right\rangle_{\mathbf{k}}$ & $0.899 \pm 0.009$ \\
$|I I\rangle_{c}|H V\rangle_{\pi}$ & & $\left|\psi^{-}\right\rangle_{\mathbf{k}}$ & $0.858 \pm 0.017$ \\
\hline$|++\rangle_{\pi}|r \ell\rangle_{\mathbf{k}}$ & $E / I$ & $\left|\phi^{+}\right\rangle_{c}$ & $0.797 \pm 0.015$ \\
\hline \hline
\end{tabular}

\footnotetext{
${ }^{1}$ In this case the projection is performed into the states $\left|\phi_{B}\right\rangle_{\mathbf{k}}=$ $\frac{1}{\sqrt{2}}\left(e^{-i \phi_{B}}|\ell\rangle_{B}+|r\rangle_{B}\right)$ and $\left|\phi_{B}^{\perp}\right\rangle_{\mathbf{k}}=\frac{1}{\sqrt{2}}\left(e^{-i \phi_{B}}|\ell\rangle_{B}-|r\rangle_{B}\right)$
} 

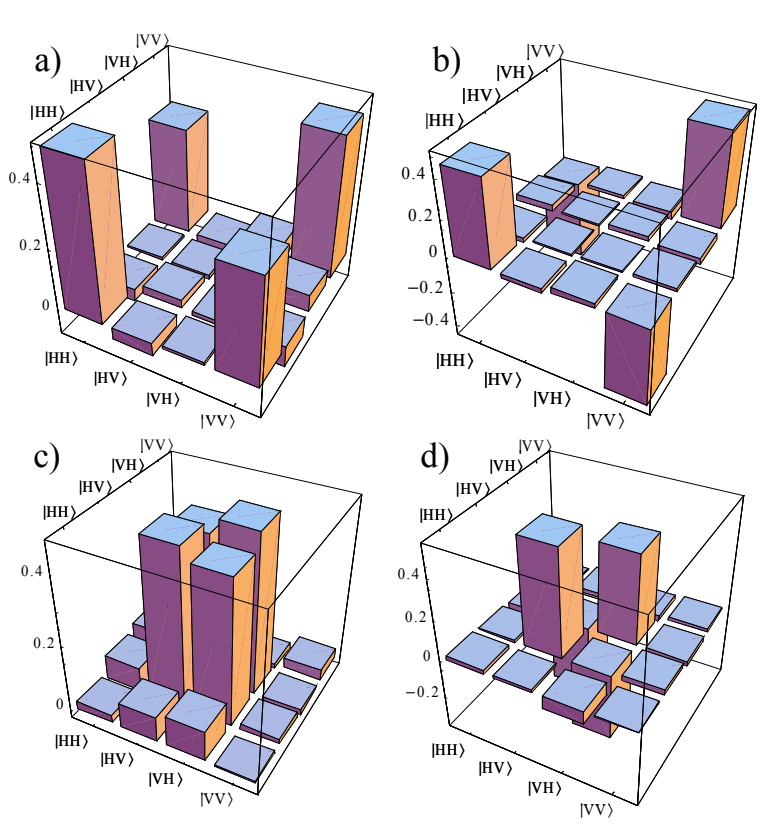

FIG. 3: Tomographic reconstruction of the four polarization states in Eq. 93 (real parts). The imaginary components are negligible. The corresponding theoretical Bell states are: (a) $\left|\Phi^{+}\right\rangle_{\pi}$, (b) $\left|\Phi^{-}\right\rangle_{\pi}$, c) $\left.\left|\Psi^{+}\right\rangle_{\pi}, \mathrm{d}\right)\left|\Psi^{-}\right\rangle_{\pi}$.
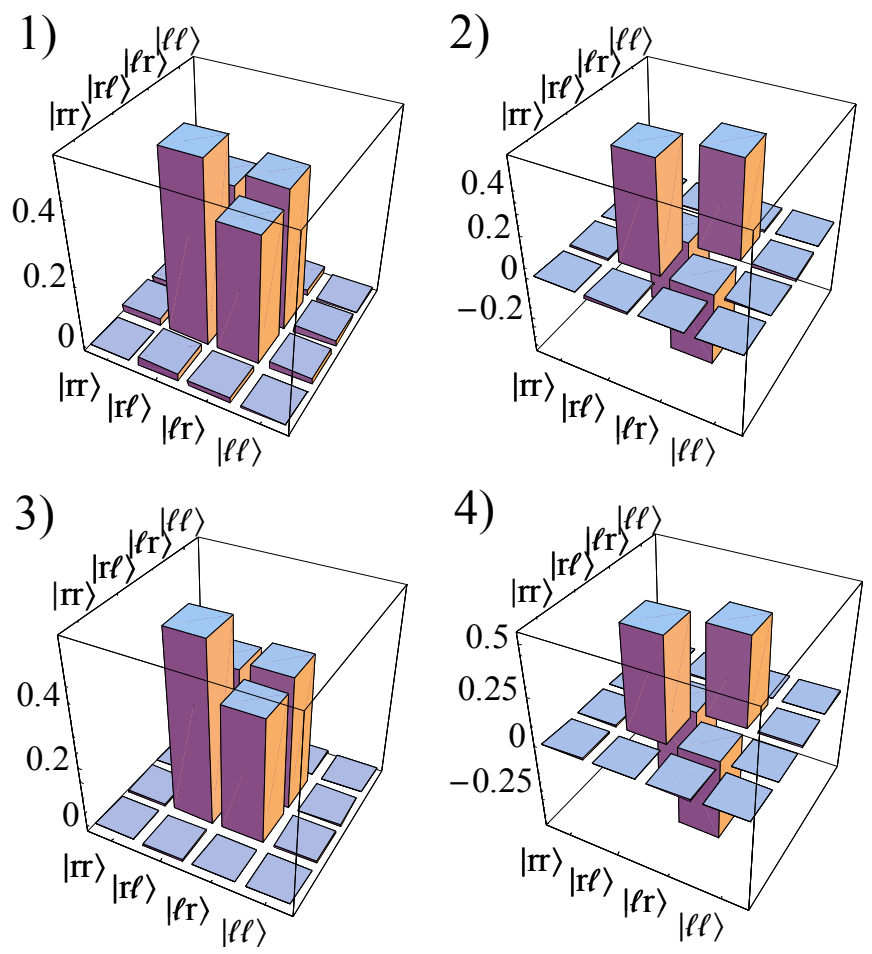

FIG. 4: Tomographic reconstruction of the four states encoded in the $r / \ell$ DOF (real parts). The imaginary components are negligible. The corresponding theoretical Bell states are: 1) and 3) $\left.\left|\Psi^{+}\right\rangle_{\mathbf{k}}, 2\right)$ and 4) $\left|\Psi^{-}\right\rangle_{\mathbf{k}}$.

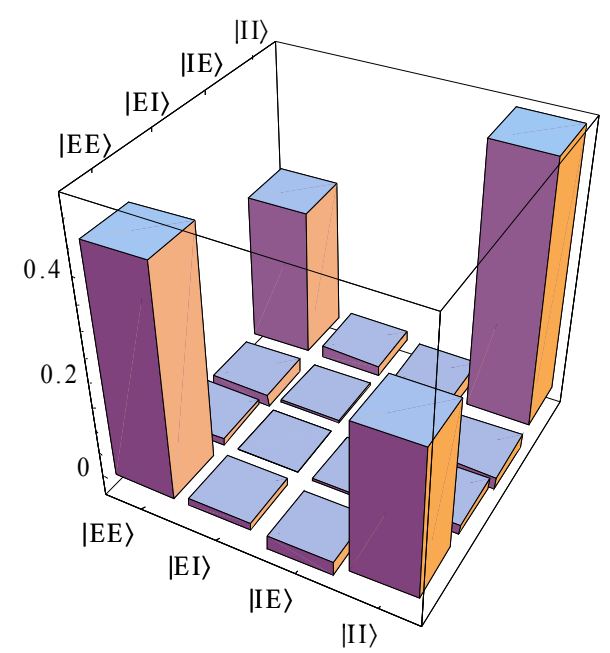

FIG. 5: Tomographic reconstruction of the state encoded in the $E / I$ DOF (real part) corresponding to the $r_{A}-\ell_{B}$ spatial mode pair and the polarization state $|+\rangle_{A}|+\rangle_{B}$. The imaginary components is negligible. The corresponding theoretical state is $\left|\phi^{+}\right\rangle_{c}$.

to two DOFs in a separable basis, and expressing the remaining couple of qubits in the appropriate entangled Bell basis; for example, the first relation shows the four polarization Bell states. Equation $9 \mathrm{a}$ ) shows that the state $\left|\widetilde{\mathrm{LC}}_{6}\right\rangle$ is obtained by a coherent superposition between four terms, each of them referring to a specific pair of correlated modes. We first demonstrated that the four polarization states corresponding to the different pairs of modes are given by the Bell states. The coherence between them can be shown by using equations $9 \mathrm{~b}$ and $9 \mathrm{c}$. It is easy to show that the first two terms in $9 \mathrm{~b}$ arise from the superposition between the first two terms in (9a), and the same applies for the last two terms. By selecting the appropriate separable basis in two DOFs we performed the tomographic reconstructions to recover the Bell states encoded in the remaining degree of freedom. As a consequence, these measurements prove not only the presence of the various terms appearing in Eq. (9), but also implicitly tell us about the coherences between the states involved.

The reconstruction concerning the polarization variable exactly followed the strategy presented in [30], while the complete sets of tomographic analysis states associated to the two longitudinal momentum DOFs were established combining the known complete set of polarization states (as given in [30]) with the stated correspondence between physical and computational qubits (see equations (6)).

The experimental density matrix reconstructions are shown in Fig. 3 for the polarization variable, in Fig. 4 for the linear momentum $\mathbf{k}$ and in Fig. 5 for the $E / I$ DOF. The fidelities associated to the considered tomographic analysis are listed in Table I. As we see, most of these values exceed $80 \%$ and some get above $90 \%$; the lowest experimental fidelity corresponds to the tomographic reconstruction associated to the $E / I$ DOF.

We attribute this to the difficulty to achieve perfect mode matching in the second interferometer due to mode diver- 
TABLE II: Experimental results: measurement of the 64 stabilizers $\widetilde{s}_{i}$ of $\left|\widetilde{\mathrm{LC}}_{6}\right\rangle$, i.e., all the products of the generators $\widetilde{g}_{i}$. Last three columns indicate in which Bell inequality test each experimental value was used.

\begin{tabular}{|ccccc|}
\hline \hline Stabilizer & Experimental value & $\mathcal{B}_{\text {exp }}$ & $\beta$ & $\beta^{\prime}$ \\
\hline 1 & $1.0000 \pm 0.0000$ & & \\
$\tilde{g}_{1}$ & $0.5928 \pm 0.0075$ & & $\checkmark$ \\
$\tilde{g}_{2}$ & $0.8788 \pm 0.0053$ & & \\
$\tilde{g}_{3}$ & $0.9984 \pm 0.0005$ & & \\
$\tilde{g}_{4}$ & $0.9973 \pm 0.0008$ & & \\
$\tilde{g}_{5}$ & $0.7905 \pm 0.0057$ & & \\
$\tilde{g}_{6}$ & $0.8310 \pm 0.0062$ & & $\checkmark$ \\
$\tilde{g}_{1} \tilde{g}_{2}$ & $0.5657 \pm 0.0059$ & & $\checkmark$ \\
$\tilde{g}_{1} \tilde{g}_{3}$ & $0.5930 \pm 0.0075$ & & \\
$\tilde{g}_{1} \tilde{g}_{4}$ & $0.5602 \pm 0.0076$ & & $\checkmark$ \\
$\tilde{g}_{1} \tilde{g}_{5}$ & $0.5872 \pm 0.0076$ & & \\
$\tilde{g}_{1} \tilde{g}_{6}$ & $0.4653 \pm 0.0095$ & $\checkmark$ & \\
$\tilde{g}_{2} \tilde{g}_{3}$ & $0.8586 \pm 0.0062$ & & \\
$\tilde{g}_{2} \tilde{g}_{4}$ & $0.8775 \pm 0.0053$ & & \\
$\tilde{g}_{2} \tilde{g}_{5}$ & $0.7042 \pm 00066$ & & \\
$\tilde{g}_{2} \tilde{g}_{6}$ & $0.8288 \pm 0.0062$ & & \\
$\tilde{g}_{3} \tilde{g}_{4}$ & $0.9970 \pm 0.0009$ & & \\
$\tilde{g}_{3} \tilde{g}_{5}$ & $0.7896 \pm 0.0057$ & & \\
$\tilde{g}_{3} \tilde{g}_{6}$ & $0.7484 \pm 0.0056$ & & $\checkmark$ \\
$\tilde{g}_{4} \tilde{g}_{5}$ & $0.7339 \pm 0.0084$ & & \\
$\tilde{g}_{4} \tilde{g}_{6}$ & $0.8312 \pm 0.0062$ & & \\
$\tilde{g}_{5} \tilde{g}_{6}$ & $0.6392 \pm 0.0060$ & & $\checkmark$ \\
$\tilde{g}_{1} \tilde{g}_{2} \tilde{g}_{3}$ & $0.4504 \pm 0.0092$ & & \\
$\tilde{g}_{1} \tilde{g}_{2} \tilde{g}_{4}$ & $0.6063 \pm 0.0074$ & & $\checkmark$ \\
$\tilde{g}_{1} \tilde{g}_{2} \tilde{g}_{5}$ & $0.5378 \pm 0.0086$ & & \\
$\tilde{g}_{1} \tilde{g}_{2} \tilde{g}_{6}$ & $0.4169 \pm 0.0065$ & $\checkmark$ & \\
$\tilde{g}_{1} \tilde{g}_{3} \tilde{g}_{4}$ & $0.5603 \pm 0.0076$ & & \\
$\tilde{g}_{1} \tilde{g}_{3} \tilde{g}_{5}$ & $0.5874 \pm 0.0075$ & & \\
$\tilde{g}_{1} \tilde{g}_{3} \tilde{g}_{6}$ & $0.4651 \pm 0.0063$ & $\checkmark$ & \\
$\tilde{g}_{1} \tilde{g}_{4} \tilde{g}_{5}$ & $0.5882 \pm 0.0074$ & & \\
$\tilde{g}_{1} \tilde{g}_{4} \tilde{g}_{6}$ & $0.4148 \pm 0.0075$ & $\checkmark$ & \\
$\tilde{g}_{1} \tilde{g}_{5} \tilde{g}_{6}$ & $0.4450 \pm 0.0061$ & $\checkmark$ & \\
\hline \hline
\end{tabular}

gences. Nevertheless, the obtained results represent a first evidence of the correct generation of the cluster state $\left|\widetilde{\mathrm{LC}}_{6}\right\rangle$. We also measured the state fidelity to give further informations on the state preparation.

As said, the reported tomographic reconstructions allow us to test the validity of Eq. (9); this approach is naturally connected to the first definition of cluster states recalled in this paper (see Eq. (2)).

We can then refer to Eq. (4) instead, which gives the characterization of cluster states in terms of their stabilizer generators, and adopt a complementary point of view (with respect to the one condensed in Eq. (97) leading to a more complete characterization of the cluster state $\left|\widetilde{\mathrm{LC}}_{6}\right\rangle$. Actually, its stabilizer generators $\left\{\tilde{g}_{i}\right\}_{i=1}^{6}$ generate the so-called stabilizer

\begin{tabular}{|ccc|}
\hline \hline Stabilizer & Experimental value & $\mathcal{B}_{\text {exp }} \beta \beta^{\prime}$ \\
\hline$\tilde{g}_{2} \tilde{g}_{3} \tilde{g}_{4}$ & $0.8592 \pm 0.0062$ & \\
$\tilde{g}_{2} \tilde{g}_{3} \tilde{g}_{5}$ & $0.7036 \pm 0.0066$ & \\
$\tilde{g}_{2} \tilde{g}_{3} \tilde{g}_{6}$ & $0.7468 \pm 0.0056$ & \\
$\tilde{g}_{2} \tilde{g}_{4} \tilde{g}_{5}$ & $0.7038 \pm 0.0066$ & \\
$\tilde{g}_{2} \tilde{g}_{4} \tilde{g}_{6}$ & $0.8285 \pm 0.0062$ & \\
$\tilde{g}_{2} \tilde{g}_{5} \tilde{g}_{6}$ & $0.6861 \pm 0.0058$ & \\
$\tilde{g}_{3} \tilde{g}_{4} \tilde{g}_{5}$ & $0.7357 \pm 0.0083$ & \\
$\tilde{g}_{3} \tilde{g}_{4} \tilde{g}_{6}$ & $0.7484 \pm 0.0056$ & \\
$\tilde{g}_{3} \tilde{g}_{5} \tilde{g}_{6}$ & $0.6625 \pm 0.0051$ & \\
$\tilde{g}_{4} \tilde{g}_{5} \tilde{g}_{6}$ & $0.6394 \pm 0.0060$ & \\
$\tilde{g}_{1} \tilde{g}_{2} \tilde{g}_{3} \tilde{g}_{4}$ & $0.6067 \pm 0.0074$ & \\
$\tilde{g}_{1} \tilde{g}_{2} \tilde{g}_{3} \tilde{g}_{5}$ & $0.5391 \pm 0.0086$ & \\
$\tilde{g}_{1} \tilde{g}_{2} \tilde{g}_{3} \tilde{g}_{6}$ & $0.4334 \pm 0.0063$ & $\checkmark$ \\
$\tilde{g}_{1} \tilde{g}_{2} \tilde{g}_{4} \tilde{g}_{5}$ & $0.4247 \pm 0.0093$ & \\
$\tilde{g}_{1} \tilde{g}_{2} \tilde{g}_{4} \tilde{g}_{6}$ & $0.3960 \pm 0.0077$ & $\checkmark$ \\
$\tilde{g}_{1} \tilde{g}_{2} \tilde{g}_{5} \tilde{g}_{6}$ & $0.4435 \pm 0.0076$ & $\checkmark$ \\
$\tilde{g}_{1} \tilde{g}_{3} \tilde{g}_{4} \tilde{g}_{5}$ & $0.5897 \pm 0.0074$ & \\
$\tilde{g}_{1} \tilde{g}_{3} \tilde{g}_{4} \tilde{g}_{6}$ & $0.4349 \pm 0.0080$ & $\checkmark$ \\
$\tilde{g}_{1} \tilde{g}_{3} \tilde{g}_{5} \tilde{g}_{6}$ & $0.4465 \pm 0.0061$ & $\checkmark$ \\
$\tilde{g}_{1} \tilde{g}_{4} \tilde{g}_{5} \tilde{g}_{6}$ & $0.4465 \pm 0.0061$ & $\checkmark$ \\
$\tilde{g}_{2} \tilde{g}_{3} \tilde{g}_{4} \tilde{g}_{5}$ & $0.7037 \pm 0.0066$ & \\
$\tilde{g}_{2} \tilde{g}_{3} \tilde{g}_{4} \tilde{g}_{6}$ & $0.7465 \pm 0.0056$ & \\
$\tilde{g}_{2} \tilde{g}_{3} \tilde{g}_{5} \tilde{g}_{6}$ & $0.6113 \pm 0.0063$ & \\
$\tilde{g}_{2} \tilde{g}_{4} \tilde{g}_{5} \tilde{g}_{6}$ & $0.6860 \pm 0.0058$ & \\
$\tilde{g}_{3} \tilde{g}_{4} \tilde{g}_{5} \tilde{g}_{6}$ & $0.6624 \pm 0.0051$ & \\
$\tilde{g}_{1} \tilde{g}_{2} \tilde{g}_{3} \tilde{g}_{4} \tilde{g}_{5}$ & $0.4235 \pm 0.0093$ & \\
$\tilde{g}_{1} \tilde{g}_{2} \tilde{g}_{3} \tilde{g}_{4} \tilde{g}_{6}$ & $0.3735 \pm 0.0078$ & $\checkmark$ \\
$\tilde{g}_{1} \tilde{g}_{2} \tilde{g}_{3} \tilde{g}_{5} \tilde{g}_{6}$ & $0.4071 \pm 0.0077$ & $\checkmark$ \\
$\tilde{g}_{1} \tilde{g}_{2} \tilde{g}_{4} \tilde{g}_{5} \tilde{g}_{6}$ & $0.5059 \pm 0.0052$ & $\checkmark$ \\
$\tilde{g}_{1} \tilde{g}_{3} \tilde{g}_{4} \tilde{g}_{5} \tilde{g}_{6}$ & $0.4884 \pm 0.0057$ & $\checkmark$ \\
$\tilde{g}_{2} \tilde{g}_{3} \tilde{g}_{4} \tilde{g}_{5} \tilde{g}_{6}$ & $0.6112 \pm 0.0063$ & \\
$\tilde{g}_{1} \tilde{g}_{2} \tilde{g}_{3} \tilde{g}_{4} \tilde{g}_{5} \tilde{g}_{6}$ & $0.4046 \pm 0.0060$ & $\checkmark$ \\
\hline \hline & &
\end{tabular}

group

$$
S\left(\left|\widetilde{\mathrm{LC}}_{6}\right\rangle\right)=\left\{S_{j}, j=1, \ldots, 2^{6}=64\right\}, \quad S_{j}=\prod_{i \in I_{j}(G)} \tilde{g}_{i}
$$

where $I_{j}(G)$ is a subset of $\{1, \cdots, 6\}$. The 64 elements $\left\{S_{j}\right\}$ are known as the stabilizing operators of $\left|\widetilde{\mathrm{LC}}_{6}\right\rangle$, and satisfy the relation $S_{j}\left|\widetilde{\mathrm{LC}}_{6}\right\rangle=\left|\widetilde{\mathrm{LC}}_{6}\right\rangle \quad \forall j=1, \ldots, 64$.

It can be shown that

$$
\left|\widetilde{\mathrm{LC}}_{6}\right\rangle\left\langle\widetilde{\mathrm{LC}}_{6}\right|=\frac{1}{64} \sum_{j=1}^{64} S_{j}
$$

The fidelity of the experimental cluster state, whose density 
matrix is $\rho_{\text {exp }}$, can then be calculated as

$$
F_{\left|\widetilde{\mathrm{LC}}_{6}\right\rangle}=\operatorname{Tr}\left[\rho_{\exp }\left|\widetilde{\mathrm{LC}}_{6}\right\rangle\left\langle\widetilde{\mathrm{LC}}_{6}\right|\right]=\frac{1}{64} \sum_{j=1}^{64} \operatorname{Tr}\left[\rho_{\exp } S_{j}\right],
$$

i.e., by measuring the expectation values of the stabilizing operators of the generated cluster state. We obtained $F_{\left|\widetilde{\mathrm{LC}}_{6}\right\rangle}=0.6350 \pm 0.0008$. The experimental expectation values $\left\{\left\langle S_{j}\right\rangle\right\}_{j=1}^{64}$ are shown in Table $\llbracket$.

We tested the genuine six-qubit entanglement of the created cluster state by evaluation of an appropriate entanglement witness, defined as [31]

$$
\mathcal{W}_{F}=\mathbb{1}-2\left|\widetilde{\mathrm{LC}}_{6}\right\rangle\left\langle\widetilde{\mathrm{LC}}_{6}\right|=\mathbb{1}-\frac{1}{32} \sum_{j=1}^{64} S_{j}
$$

There is entanglement whenever

$$
\left\langle\mathcal{W}_{F}\right\rangle=\mathbb{1}-2 F<0 .
$$

We found $\left\langle\mathcal{W}_{F}\right\rangle=-0.270 \pm 0.002$, which being negative by 135 standard deviations proves the existence of a genuine six-qubit entanglement.

The data present in Table test of quantum mechanics [29]. Any local theory in which every single-qubit Pauli observable can be interpreted as an element of reality as intended by EPR satisfies the following inequality:

$$
\mathcal{B} \leq 4 \equiv B_{\mathrm{LHVT}},
$$

where $\mathcal{B}$ is defined as

$$
\mathcal{B}=\left|g_{1}\left(\mathbb{1}+g_{2}\right)\left(\mathbb{1}+g_{3}\right)\left(\mathbb{1}+g_{4}\right)\left(\mathbb{1}+g_{5}\right) g_{6}\right| .
$$

We tested the Bell inequality (15) and obtained $\mathcal{B}_{\exp }=$ $7.018 \pm 0.028$ (see checked rows in the third column of Table II); this result implies a degree of nonlocality $\mathcal{D}=\frac{\mathcal{B}_{\text {exp }}}{B_{\mathrm{LHVT}}}$ equal to $1.7545 \pm 0.0070$. We also tested the persistency of entanglement of $\left|\widetilde{\mathrm{LC}}_{6}\right\rangle$ against the loss of two qubits. This property can be investigated by considering two alternative Bell inequalities with respect to (15): in the first one qubits 3 and 6 are ignored, while in the second inequality we trace out qubits 1 and 4:

$$
\begin{aligned}
& \beta=\left|g_{1}\left(\mathbb{1}+g_{2}\right)\left(\mathbb{1}+g_{4}\right)\right| \stackrel{L H V}{\leq} 2, \\
& \beta^{\prime}=\left|\left(\mathbb{1}+g_{3}\right)\left(\mathbb{1}+g_{5}\right) g_{6}\right| \stackrel{L H V}{\leq} 2
\end{aligned}
$$

By using the measurements given in Table 1 we found

$$
\begin{aligned}
& \beta_{\exp }=2.325 \pm 0.014, \\
& \beta_{\exp }^{\prime}=2.881 \pm 0.012,
\end{aligned}
$$

showing violations of the Bell inequalities (17a) and 17b). See [29] for more details concerning Bell inequalities with the 2-photon 6-qubit cluster state.

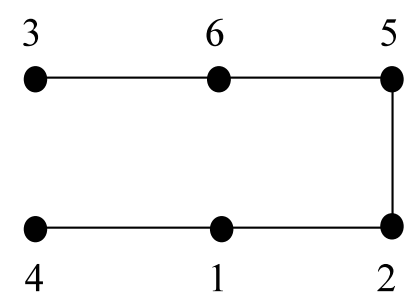

FIG. 6: Graph associated to the six-qubit horseshoe cluster state, equivalent to the generated linear cluster state.

\section{EXPERIMENTAL REALIZATION OF THE CNOT GATE}

Let us now turn to the one-way model of QC [10]. Given a cluster state, it can be useful to think of the distinct horizontal qubits as "the original [logical] qubit at different times" [32], with the temporal axis oriented from left to right (a choice made possible by appropriately designing the lattice); singlequbit gates are represented by pairs of horizontally adjacent qubits, while vertical connections play the role of CPHASE gates. Each computation process is then obtained as a sequence of single-qubit projective measurements performed on the so-called physical qubits, simultaneously determining the propagation of information through the cluster and the loss of entanglement in the original state [6, 32].

This last feature is responsible for the irreversibility of the process and explains why we speak of one-way computation. The difference existing between physical and encoded qubits deserves a deeper understanding. Physical qubits in the initial cluster state represent an entanglement resource; encoded (or logical) qubits constitute the quantum information being processed [33]. Let $N$ be the number of physical qubits and $M$ the number of encoded qubits, with $M<N . M$ input cluster qubits, all prepared in the state $|+\rangle$, are usually positioned on the left of the two-dimensional graph. The single-qubit measurements involve $N-M$ qubits. Consequently, the output of the computation can be read on the $M$ unmeasured qubits up to local Pauli errors, as will be specified later on in this paper. More precisely, the measurements driving the computation are performed in the following basis:

$$
B_{i}(\alpha)=\left\{\left|\alpha_{+}\right\rangle_{i},\left|\alpha_{-}\right\rangle_{i}\right\},
$$

with $\left|\alpha_{ \pm}\right\rangle_{i}=\frac{1}{\sqrt{2}}\left(e^{i \alpha / 2}|0\rangle_{i}+e^{-i \alpha / 2}|1\rangle_{i}\right)$. If we take $s_{i}$ as signalling the presence of a Pauli error, we usually associate $s_{i}=0$ to the measurement outcome $\left|\alpha_{+}\right\rangle$(error-free case) and $s_{i}=1$ to $\left|\alpha_{-}\right\rangle$. The choice of $\alpha$ (and the consequent possible errors occurring in the computation) depends on the algorithm to be implemented. Measuring a qubit in the computational basis $\left\{|0\rangle_{i},|1\rangle_{i}\right\}$ has a completely different effect on the cluster, in that it removes the measured qubit and leads to the cluster state

$$
\prod_{k \in \mathcal{N}_{i}} Z_{k}^{s_{i}}\left|\Phi_{N-1}^{\mathcal{L} \backslash\{i\}}\right\rangle
$$




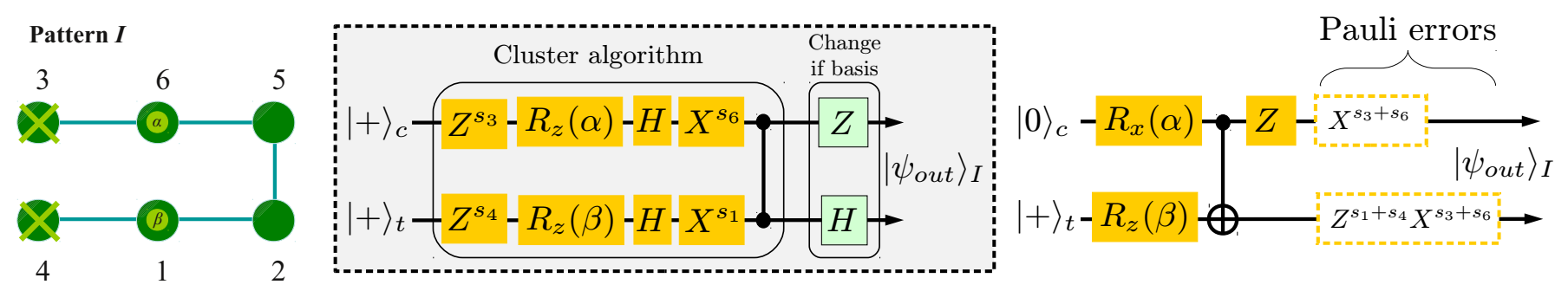

FIG. 7: Measurement pattern I: we indicate by a cross a measurement in the bases $\{|0\rangle,|1\rangle\}$ and by $\alpha$ and $\beta$ a measurement in the basis $B(\alpha)$ and $B(\beta)$. The output state is encoded in qubits 5 and 2. The circuit associated to the considered measurement is shown. We first indicate the circuit obtained by directly following the one-way rules and then the equivalent circuit composed by single-qubit gates and a two-qubit CNOT gate. The gates indicated by "Change of basis" are due to the change between the computational and laboratory basis.

\begin{tabular}{|clcc|}
\hline \hline Pattern & Qubit [DOF] & Measurement CB & Measurement LB \\
\hline$I$ & $3[r / \ell]$ & $\{|0\rangle,|1\rangle\}$ & $\{|+\rangle,|-\rangle\}$ \\
& $4[E / I]$ & $\{|0\rangle,|1\rangle\}$ & $\{|+\rangle,|-\rangle\}$ \\
& $6[r / \ell]$ & $B(\alpha)^{a}$ & $B(\alpha)^{a}$ \\
& $1[E / I]$ & $B(\beta)^{a}$ & $B(\beta)^{a}$ \\
\hline$I I$ & $3[r / \ell]$ & $\{|0\rangle,|1\rangle\}$ & $\{|+\rangle,|-\rangle\}$ \\
& $4[E / I]$ & $B(0)$ & $\{|0\rangle,|1\rangle\}$ \\
& $6[r / \ell]$ & $B(\alpha)$ & $B(\alpha)$ \\
& $1[E / I]$ & $B(0)$ & $\{|+\rangle,|-\rangle\}$ \\
\hline$I I I$ & $3[r / \ell]$ & $B(0)$ & $\{|1\rangle,|0\rangle\}$ \\
& $4[E / I]$ & $\{|0\rangle,|1\rangle\}$ & $\{|+\rangle,|-\rangle\}$ \\
& $6[r / \ell]$ & $B(0)$ & $\{|+\rangle,|-\rangle\}$ \\
& $1[E / I]$ & $B(\beta)$ & $B(\beta)$ \\
\hline$I V$ & $3[r / \ell]$ & $B(0)$ & $\{|1\rangle,|0\rangle\}$ \\
& $4[E / I]$ & $B(0)$ & $\{|0\rangle,|1\rangle\}$ \\
& $6[r / \ell]$ & $B(0)$ & $\{|+\rangle,|-\rangle\}$ \\
& $1[E / I]$ & $B(0)$ & $\{|+\rangle,|-\rangle\}$ \\
\hline \hline
\end{tabular}

TABLE III: Measurement bases for the different considered patterns. For each pattern we indicate the measured qubit (and the DOF in which the qubit is encoded) and the corresponding measurement in the cluster (CB) and laboratory bases (LB).

${ }^{a}$ See Eq. 19.

where $\mathcal{N}_{i}$ is the set of vertices connected to site $i$.

The generated six-qubit cluster allows the implementation of non-trivial two-qubit operations such as the CNOT gate. For this purpose, it is convenient to think of a horseshoe $\left(180^{\circ}\right.$ rotated) six-qubit cluster instead of the one depicted in Fig \b); the two are physically equivalent, but the horseshoe one reveals easier to translate into a circuit representation of the CNOT gate. Let us consider Fig. 6 Since we realize our computation within the one-way model, we perform simultaneous single-qubit measurements on qubits 3 and 4 and on qubits 6 and 1 and then read the corresponding output on qubits 5 and 2 , both encoded in the polarization DOF.

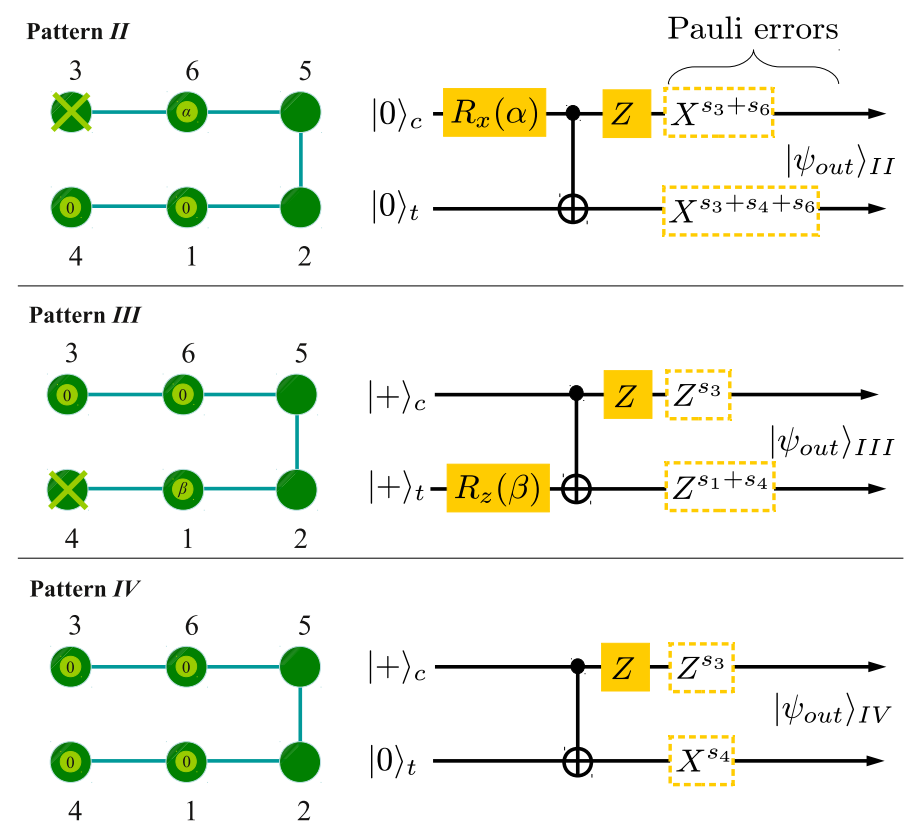

FIG. 8: Measurement patterns $I I, I I I$ and $I V$ and the corresponding circuit representations. Each circuit is composed by single-qubit gates and a two-qubit CNOT gate.

We pointed out four possible measurement patterns in order to accomplish different logical operations, depending on the bases chosen for the single-qubit measurements. From now on, when referring to a given measurement basis we will always think of the so-called "laboratory basis" (LB), which differs from the "cluster basis" $(\mathrm{CB})$ because of the presence of the local operations affecting qubits $3\left(X_{3} \mathrm{H}_{3}\right)$ and $4\left(\mathrm{H}_{4}\right)$ (see Eq. (8)). The four considered measurement patterns, both in the cluster and in the laboratory bases, are listed in Table

For each pattern a corresponding computational circuit can be derived. In Fig. 7 we show the detailed derivation of the corresponding circuit for the first considered pattern: the measurements implement the "Cluster algorithm" (see figure) and 
the change between the $\mathrm{CB}$ and the $\mathrm{LB}$ corresponds to the final gates (labeled as "Change of basis" in the figure). The circuit can be equivalently written as shown in the right part: it consists of two single qubit rotations and a CNOT gate. The Pauli errors, as usual, depend on the measurement results of qubits 3, 4, 6 and 1. In Fig. 8 we show the equivalent circuits corresponding to the other three measurement patterns we have considered.

By taking into account their circuit representations shown in Fig. 7 and Fig. 8 we can write the expected output state, encoded in the physical qubits 5 (photon $B$, control) and 2 (photon $A$, target), for each measurement pattern:

$$
\begin{aligned}
\left|\psi_{\text {out }}\right\rangle_{I}= & \left(X_{5}^{s_{3}+s_{6}} Z_{2}^{s_{1}+s_{4}} X_{2}^{s_{3}+s_{6}}\right) \times \\
& \times Z_{5} \mathrm{CNOT}_{52}\left[R_{x}^{(5)}(\alpha) \otimes R_{z}^{(2)}(\beta)\right]|0\rangle_{5}|+\rangle_{2} \\
\left|\psi_{\text {out }}\right\rangle_{I I}= & \left(X_{5}^{s_{3}+s_{6}} X_{2}^{s_{3}+s_{4}+s_{6}}\right) \times \\
& \times Z_{5} \mathrm{CNOT}_{52}\left[R_{x}^{(5)}(\alpha) \otimes \mathbb{1}_{2}\right]|0\rangle_{5}|0\rangle_{2} \\
\left|\psi_{\text {out }}\right\rangle_{I I I}= & \left(Z_{5}^{s_{3}} Z_{2}^{s_{1}+s_{4}}\right) \times \\
& \times Z_{5} \mathrm{CNOT}_{52}\left[\mathbb{1}_{5} \otimes R_{z}^{(2)}(\beta)\right]|+\rangle_{5}|+\rangle_{2} \\
\left|\psi_{\text {out }}\right\rangle_{I V}= & \left(Z_{B}^{s_{3}} X_{A}^{s_{4}}\right) Z_{5} \mathrm{CNOT}_{52}|+\rangle_{5}|0\rangle_{2}
\end{aligned}
$$

where $R_{x}(\alpha)=e^{-i \alpha X / 2}$ corresponds to a counterclockwise rotation through an angle $\alpha$ about the $x$ axis of the Bloch sphere (an analogous definition holds for $R_{z}(\beta)$ ). The presenve of the single-qubit Pauli errors $X$ and $Z$ depends on the measurement output of the corresponding qubit (represented by $s_{i}$ for $i=1,3,4,6$, see the comment following Eq. (19p). It comes out from the expressions of $\left|\psi_{\text {out }}\right\rangle$ in (21) that the computations can be interpreted as single qubit transformations followed by a CNOT gate acting on different input states. Precisely, by rewriting the computation in the error-free case we obtain:

$$
\begin{aligned}
\left|\psi_{\text {out }}\right\rangle_{I} & =Z_{5} \operatorname{CNOT}_{52}\left[R_{x}^{(5)}(\alpha) \otimes R_{z}^{(2)}(\beta)\right]\left|\psi_{\text {in }}\right\rangle_{I}, \\
\left|\psi_{\text {out }}\right\rangle_{I I} & =Z_{5} \operatorname{CNOT}_{52}\left[R_{x}^{(5)}(\alpha) \otimes \mathbb{1}_{2}\right]\left|\psi_{\text {in }}\right\rangle_{I I}, \\
\left|\psi_{\text {out }}\right\rangle_{I I I} & =Z_{5} \operatorname{CNOT}_{52}\left[\mathbb{1}_{5} \otimes R_{z}^{(2)}(\beta)\right]\left|\psi_{\text {in }}\right\rangle_{I I I}, \\
\left|\psi_{\text {out }}\right\rangle_{I V} & =Z_{5} \operatorname{CNOT}_{52}\left|\psi_{\text {in }}\right\rangle_{I V}
\end{aligned}
$$

where the input states are $\left|\psi_{\text {in }}\right\rangle_{I}=|0\rangle_{5}|+\rangle_{2},\left|\psi_{\text {in }}\right\rangle_{I I}=$ $|0\rangle_{5}|0\rangle_{2},\left|\psi_{\text {in }}\right\rangle_{I I I}=|+\rangle_{5}|+\rangle_{2}$ and $\left|\psi_{\text {in }}\right\rangle_{I V}=|+\rangle_{5}|0\rangle_{2}$.

Let's start from pattern $I V$ : in this case, by looking at the measurement basis given in Table III, it is possible to reinterpret the four tomographic reconstructions of the cluster state $\left|\widetilde{\mathrm{LC}}_{6}\right\rangle$ with respect to the polarization DOF as a one-way computation (here the CNOT operation). Precisely, the measurement of qubits 3 and 4 in the computational basis corresponds to selecting different modes of the cluster. The output is then encoded in the polarization of the two photons and the four Bell states correspond to the four different outputs of the computation. In fact, it is easy to show that $\left|\psi_{\text {out }}\right\rangle_{I V}=\left|\phi^{-}\right\rangle_{52}$ in the error-free case. The other three Bell states are obtained by applying the different Pauli errors. Hence the tomographic reconstructions of the polarization states given in Sec [II] suffice to the experimental proof of the correct functioning of the realized logic gate within the specific framework of pattern $I V$.
TABLE IV: Input-output states, corresponding to the first three measurement patterns, expressed in the polarization basis. Here the "AB" ordering is used.

\begin{tabular}{cccc|}
\hline \hline Pattern & Input state $^{b}$ & Expected output state $^{b}$ & Fidelity \\
\hline$I$ & $|+H\rangle_{A B}$ & $|+H\rangle_{A B}$ & $0.6052 \pm 0.0084$ \\
& $|-H\rangle_{A B}$ & $|-H\rangle_{A B}$ & $0.6657 \pm 0.0077$ \\
& $|-V\rangle_{A B}$ & $|-V\rangle_{A B}$ & $0.5476 \pm 0.0066$ \\
& $|+V\rangle_{A B}$ & $|+V\rangle_{A B}$ & $0.6223 \pm 0.0069$ \\
\hline$I I$ & $|H H\rangle_{A B}$ & $|H H\rangle_{A B}$ & $0.8716 \pm 0.0050$ \\
& $|V H\rangle_{A B}$ & $|V H\rangle_{A B}$ & $0.8348 \pm 0.0072$ \\
& $|H V\rangle_{A B}$ & $|V V\rangle_{A B}$ & $0.8710 \pm 0.0053$ \\
& $|V V\rangle_{A B}$ & $|H V\rangle_{A B}$ & $0.8376 \pm 0.0065$ \\
\hline$I I I$ & $|++\rangle_{A B}$ & $|+-\rangle_{A B}$ & $0.6541 \pm 0.0111$ \\
& $|+-\rangle_{A B}$ & $|++\rangle_{A B}$ & $0.6798 \pm 0.0088$ \\
& $|--\rangle_{A B}$ & $|--\rangle_{A B}$ & $0.6741 \pm 0.0108$ \\
& $|-+\rangle_{A B}$ & $|-+\rangle_{A B}$ & $0.6096 \pm 0.0093$ \\
\hline \hline
\end{tabular}

Let's then consider patterns $I, I I$ and $I I I$ in the error-free case, which means that $s_{i}=0$ for $i=1,3,4,6$. Moreover we set $\alpha=\beta=0$, implying that $R_{x}(0)=R_{z}(0)=\mathbb{1}$. These hypothesis lead to the output states $\left|\psi_{\text {out }}\right\rangle_{I},\left|\psi_{\text {out }}\right\rangle_{I I}$ and $\left|\psi_{\text {out }}\right\rangle_{I I I}$, all in the form of separable states of the two photons $A$ and $B$, and establish a first set of input and output states for the three cases. In these conditions, it is interesting to reconstruct the input-output (I-O) matrices for the realized CNOT gate, whose knowledge enables the further calculation of the fidelities associated to the output states 22a (22c). The experimental results are listed in Table IV while Fig. 9 shows a graphic representation of the I-O matrices. The fidelity values show that the gate built on the generated cluster state $\left|\widetilde{\mathrm{LC}}_{6}\right\rangle$ operates as expected.

A further analysis consists of examining other possible values for the rotation angles $\alpha$ and $\beta$ in the framework of an error-free computation. By letting $\alpha$ and $\beta$ assume non-zero values during the computation, we obtain other combinations of input and output product states. As an example, we consider the "variant" of pattern $I I$ where $\alpha=3 \pi / 2$. We can then write the output state as

$$
\begin{aligned}
\left|\psi_{\text {out }}^{\prime}\right\rangle_{I I} & =Z_{5} \operatorname{CNOT}_{52} R_{x}^{(5)}(3 \pi / 2)|0\rangle_{5}|0\rangle_{2}= \\
& =-\frac{1}{\sqrt{2}}\left(|H H\rangle_{A B}-i|V V\rangle_{A B}\right) .
\end{aligned}
$$

As we see, here we have an entangled two-photon state encoded in polarization. When dealing with an entangled state of photons $\mathrm{A}$ and $\mathrm{B}$ it is not possible to adopt an I-O matrix reconstruction strategy in order to test the correctness of the gate's functioning; it is now necessary to perform a tomographic reconstruction of the output state corresponding to the considered computation (this is exactly what happens with case $I V$, too). The experimental tomographic analysis for the "case $I I$ variant" is shown in Fig. 10, the fidelity of the output state $\left|\psi_{\text {out }}^{\prime}\right\rangle_{I I}$ is $F=0.879 \pm 0.017$. 

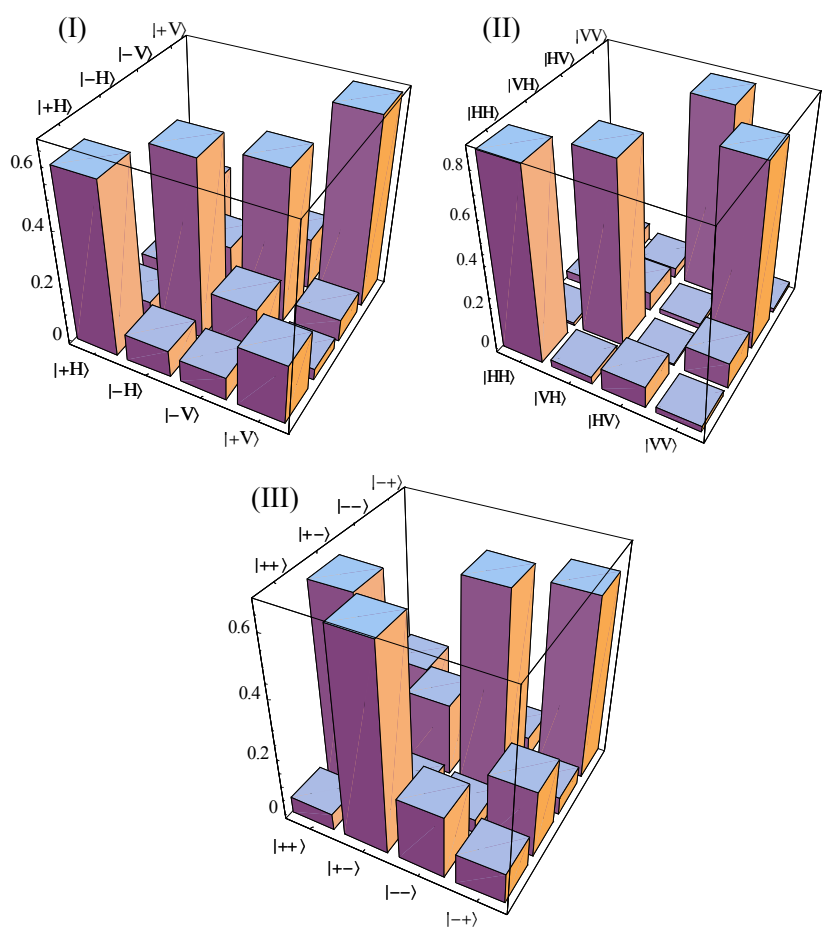

FIG. 9: Graphic representation of the I-O matrices for the considered CNOT operation. The sublabels indicate the pattern to which each matrix refers to. The sets of input and output states are listed in Table IV] and can be read on the upper (input) and lower (output) axis.
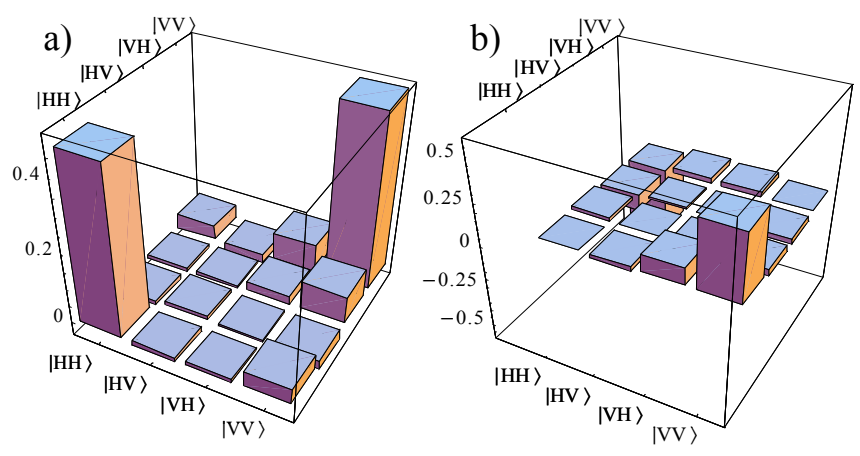

FIG. 10: Tomographic reconstruction of the polarization entangled output state $\left|\psi_{\text {out }}^{\prime}\right\rangle_{I I}$. Both the (a) real and (b) imaginary components are shown. The corresponding theoretical state is $-\frac{1}{\sqrt{2}}\left(|H H\rangle_{A B}-i|V V\rangle_{A B}\right)$.

\section{CONCLUSIONS AND PERSPECTIVES}

We have characterized the six-qubit linear cluster state $\left|\widetilde{\mathrm{LC}}_{6}\right\rangle$, realized by starting from a two-photon state, hyperentangled in the three degrees of freedom of polarization and a double set of longitudinal momentum modes of the photons emitted over the degenerate cone of a Type I SPDC crystal.

The importance of a six-qubit linear cluster state is twofold: while it represents a significant step in the research of quantum nonlocality, as recently demonstrated [29], the realization of cluster states with an increasing number of qubits is important to the quantum computation community. Indeed, we have used this state by realizing the CNOT gate in the one-way quantum computation domain. For this purpose, the configuration chosen for the graph associated to the six-qubit state is that of a horseshoe $\left(180^{\circ}\right.$ rotated) cluster state.

The CNOT results of our experiment are similar to those obtained within the Hefei's group experiment [34], where a sixqubit graph state has been created by using polarization and spatial modes of four photons. By that technique new qubits encoded in different DOFs of the same photon are added by local operations. Multi-qubit entangled states realized by this technique may find useful applications in one-way quantum computation. However, it has been already emphasized that in such states polarization and longitudinal momentum of the same photon are not independent [28]. As a consequence, regarding their use in quantum nonlocality tests, some problems may arise in the definition of EPR's criterion for elements of reality [35].

Both the existing approaches to one-way QC and error encoding, based on multi-photon and multi-DOF entanglement, contribute to make an all-optical architecture a serious contender for the ultimate goal of a large-scale quantum computer. However, scalable linear optics systems are required for the realization of more complex QC operations and algorithms. This is a very challenging objective, according to the current optical technology. One of the main reasons is that an increasing number of qubits requires the setup of bulk measurement systems of increasing complexity. At the same time, the need of an increasing number of qubits in a QC algorithm conflicts with the intrinsic limitations of the SPDC process. Indeed, no more than few pairs of photons at a time are created by SPDC, due to its probabilistic nature. Moreover, multiphoton detection is seriously affected by the limited quantum efficiencies of modern detectors.

In order to take the maximum advantage of the possibilities offered by the current optical technology to increase power and speed of computational operations based on high dimension entangled photonic systems, we may conceive cluster states built on a number of photons entangled in many DOFs. Increasing the number of photons or encoding the qubits in other DOFs of the particles, besides polarization and longitudinal momentum, such as frequency, time bin and orbital angular momentum of the photons, are two complementary (but not exclusive) approaches to enhance the computational power and the information content.

It is worth to remember that increasing the number $\mathrm{n}$ of involved DOFs implies an exponential requirement of resources. For instance, $2^{n} \mathbf{k}$-modes per photon must be selected within the emission cone to encode $n$ qubits in each photon. However, according to the current optical technology, working with few DOFs (such as $n=2,3,4$ ) offers still more advantages than working with a corresponding number of photon pairs, because of the higher repetition rate and 
state generation/detection efficiency. Indeed, by increasing the number of DOFs on which two photons are entangled, the overall detection efficiency and hence the repetition rate of detection is constant, since it scales as $\eta^{N}$, being $N$ the number of photons and $\eta$ the detector quantum efficiency, except for some factors depending on the measurement setup. Furthermore, an entangled state built on a larger number of particles is in principle more affected by decoherence because of the increased difficulty of making photons indistinguishable. In medium-term time scale a hybrid approach to QC (i.e. multi-DOF and multi-photon states) may represent a convenient solution to overcome the structural limitations in generation/detection of quantum photon states.

In view of an efficient linear optics quantum computation, the use of miniaturized optical circuits built on a chip in the realization of increasingly complex linear optical schemes consisting of many interferometers, whose feasibility has been recently demonstrated [36, 37, 38, 39], is becoming of fundamental relevance. Indeed these new integrated structures guarantee high fidelities and highly intrinsic phase stability of the measurements necessary to perform the logical operations. Furthermore, the adoption of integrated optics may also enable the realization of novel kinds of multi-photon states. Hence, new exciting perspectives implying the solution of new problems are opened in the application of miniaturized optical structures with multi-photon multi-DOF entangled states.
[1] M. Hein, J. Eisert, and H. J. Briegel, Phys. Rev. A 69, 062311 (2004).

[2] N. D. Mermin, Phys. Rev. Lett. 65, 1838 (1990).

[3] O. Gühne, G. Tóth, P. Hyllus, and H. J. Briegel, Phys. Rev. Lett. 95, 120405 (2005).

[4] A. Cabello and P. Moreno, Phys. Rev. Lett. 99, 220402 (2007).

[5] A. Cabello, O. Gühne, and D. Rodríguez, Phys. Rev. A 77, 062106 (2008).

[6] R. Raussendorf and H. J. Briegel, Phys. Rev. Lett. 86, 5188 (2001).

[7] H. J. Briegel and R. Raussendorf, Phys. Rev. Lett. 86, 910 (2001).

[8] R. Cleve, D. Gottesman, and H.-K. Lo, Phys. Rev. Lett. 83, 648 (1999).

[9] D. Schlingemann and R. F. Werner, Phys. Rev. A 65, 012308 (2001).

[10] H. J. Briegel, D. E. Browne, W. Dür, R. Raussendorf, and M. V. den Nest, Nature Physics 5, 19 (2009).

[11] K. Chen, C.-M. Li, Q. Zhang, Y.A. Chen, A. Goebel, S. Chen, A. Mair, and J.-W. Pan, Phys. Rev. Lett. 99, 120503 (2007).

[12] G. Vallone, E. Pomarico, F. De Martini, and P. Mataloni, Phys. Rev. Lett. 100, 160502 (2008).

[13] Z. Zhao, T. Yang, Y.A. Chen, A.-N. Zhang, M. Żukowski, and J.-W. Pan, Phys. Rev. Lett. 91, 180401 (2003).

[14] P. Walther, K. J. Resch, T. Rudolph, E. Schenck, H. Weinfurter, V. Vedral, M. Aspelmeyer, and A. Zeilinger, Nature (London) 434, 169 (2005)

[15] P. Walther, M. Aspelmeyer, K. J. Resch, and A. Zeilinger, Phys. Rev. Lett. 95, 020403 (2005).

[16] N. Kiesel, C. Schmid, U. Weber, G. Tóth, O. Gühne, R. Ursin, and H. Weinfurter, Phys. Rev. Lett. 95, 210502 (2005).

[17] R. Prevedel, P. Walther, F. Tiefenbacher, P. Böhi, R. Kaltenbaek, T. Jennewein, and A. Zeilinger, Nature (London) 445, 65 (2007).

[18] G. Vallone, E. Pomarico, P. Mataloni, F. De Martini, and V. Berardi, Phys. Rev. Lett. 98, 180502 (2007).

[19] W.-B. Gao, C.-Y. Lu, X.-C. Yao, P. Xu, O. Ghne, A. Goebel, Y.-A. Chen, C.-Z. Peng, Z.-B. Chen, and J.-W. Pan (2008), preprint, arXiv:0809.4277.

[20] Y. Tokunaga, S. Kuwashiro, T. Yamamoto, M. Koashi, and N. Imoto, Phys. Rev. Lett. 100, 210501 (2008).
[21] A. Mair, A. Vaziri, G. Weihs, and A. Zeilinger, Nature (London) 412, 313 (2001).

[22] C. Cinelli, M. Barbieri, R. Perris, P. Mataloni, and F. De Martini, Phys. Rev. Lett. 95, 240405 (2005).

[23] M. Barbieri, C. Cinelli, P. Mataloni, and F. De Martini, Phys. Rev. A 72, 052110 (2005).

[24] J. T. Barreiro, N. K. Langford, N. A. Peters, and P. G. Kwiat, Phys. Rev. Lett. 95, 260501 (2005).

[25] C. Schuck, G. Huber, C. Kurtsiefer, and H. Weinfurter, Phys. Rev. Lett. 96, 190501 (2006).

[26] H. S. Park, J. Cho, J. Y. Lee, D.-H. Lee, and S.-K. Choi, Opt. Express 15, 17960 (2007).

[27] B. P. Lanyon, M. Barbieri, M. P. Almeida, T. Jennewein, T. C. Ralph, K. J. Resch, G. J. Pryde, J. L. O’Brien, A. Gilchrist, and A. G. White, Nature Physics 5, 134 (2009).

[28] G. Vallone, R. Ceccarelli, F. De Martini, and P. Mataloni, Phys. Rev. A 79, 030301(R) (2009).

[29] R. Ceccarelli, G. Vallone, F. De Martini, P. Mataloni, and A. Cabello, Phys. Rev. Lett. 103, 160401 (2009).

[30] D. F. V. James, P. G. Kwiat, W. J. Munro, and A. G. White, Phys. Rev. A 64, 052312 (2001).

[31] G. Tóth and O. Gühne, Phys. Rev. Lett. 94, 060501 (2005).

[32] M. A. Nielsen, Phys. Rev. Lett. 93, 040503 (2004).

[33] R. Raussendorf, D. E. Browne, and H. J. Briegel, Phys. Rev. A 68, 022312 (2003).

[34] W.-B. Gao, P. Xu, X.-C. Yao, O. Gühne, A. Cabello, C.-Y. Lu, C.-Z. Peng, Z.-B. Chen, and J.-W. Pan (2009), preprint, arXiv:0905.2103|.

[35] W.-B. Gao, X.-C. Yao, P. Xu, O. Ghne, A. Cabello, C.Y. Lu, T. Yang, Z.-B. Chen, and J.-W. Pan, preprint, |arXiv:0906.3390|.

[36] A. Politi, M. J. Cryan, J. G. Rarity, S. Yu, and J. L. O’Brien, Science 320, 646 (2008).

[37] A. Politi, J. C. F. Matthews, and J. L. O'Brien, Science 325, 1221 (2009).

[38] J. Matthews, A. Politi, A. Stefanov, and J. L. O'Brien, Nature Photonic 3, 346 (2009).

[39] G. D. Marshall, A. Politi, J. C. F. Matthews, P. Dekker, M. Ams, M. J. Withford, and J. L. O'Brien, Optics Express 17, 12546 (2009). 